\title{
Numerical Modeling of Anisotropic Elastic-Wave Sensitivity Propagation for Optimal Design of Time-Lapse Seismic Surveys
}

\author{
Kai Gao* and Lianjie Huang \\ Los Alamos National Laboratory, Geophysics Group, MS D452, Los Alamos, \\ NM 87545, USA.
}

Received 31 August 2018; Accepted (in revised version) 2 March 2019

\begin{abstract}
Reliable subsurface time-lapse seismic monitoring is crucial for many geophysical applications, such as enhanced geothermal system characterization, geologic carbon utilization and storage, and conventional and unconventional oil/gas reservoir characterization, etc. We develop an elastic-wave sensitivity propagation method for optimal design of cost-effective time-lapse seismic surveys considering the fact that most of subsurface geologic layers and fractured reservoirs are anisotropic instead of isotropic. For anisotropic media, we define monitoring criteria using $\mathrm{qP}-$ and $\mathrm{qS}-$ wave sensitivity energies after decomposing $\mathrm{qP}$ - and qS-wave components from the total elastic-wave sensitivity wavefield using a hybrid time- and frequency-domain approach. Geophones should therefore be placed at locations with significant qPand qS-wave sensitivity energies for cost-effective time-lapse seismic monitoring in an anisotropic geology setting. Our numerical modeling results for a modified anisotropic Hess model demonstrate that, compared with the isotropic case, subsurface anisotropy changes the spatial distributions of elastic-wave sensitivity energies. Consequently, it is necessary to consider subsurface anisotropies when designing the spatial distribution of geophones for cost-effective time-lapse seismic monitoring. This finding suggests that it is essential to use our new anisotropic elastic-wave sensitivity modeling method for optimal design of time-lapse seismic surveys to reliably monitor the changes in subsurface reservoirs, fracture zones or target monitoring regions.
\end{abstract}

AMS subject classifications: (or PACs) To be provided by authors

Key words: Anisotropy, elastic-wave sensitivity, time-lapse seismic monitoring.

\section{Introduction}

The goal of time-lapse seismic monitoring is to reveal the temporal media property changes of subsurface target regions, such as fracture zones/fault zones in geologic car-

*Corresponding author. Email addresses: kaigao@lanl .gov (K. Gao), ljh@lanl .gov (L. Huang) 
bon storage reservoirs, geothermal reservoirs and fractured reservoirs. This goal is usually achieved by subtracting the imaging or inversion results of the target monitoring regions before and after a certain time interval, using the same source-receiver geometry configuration. However, the accuracy of this approach can be degraded by numerous factors, such as random environmental noises, petrophysical environment changes beyond the detection of existing techniques, data acquisition repeatability, and changes in data processing flows across time-lapse surveys. To reduce the influence of unpredictable factors and accurately extract changes in target monitoring regions, it is necessary to design a seismic source-receiver network to cost-effectively capture the most significant seismic signals from time-lapse changes in target monitoring regions.

Time-lapse seismic monitoring becomes more complicated when subsurface geologic layers and the target monitoring region are anisotropic media, such as thin layers and fracture/fault zones. Naturally occurring cracks and fractures usually exhibit preferentially spatial alignment, resulting in significant velocity and permeability anisotropies $[8-10,12,14-17,19,20]$. For example, a set of rotationally invariant fractures sharing a common symmetry axis could result in transverse isotropy along the symmetry axis, which may lie horizontally [20], and orthogonally aligned fractures may result in orthotropic anisotropy $[20,24]$. Because seismic signals reflected/scattered from these fractures significantly complicate both data acquisition and subsequent data processing flow, we cannot determine the media properties of fracture zones as reliably as those of simple geology. In addition, most sedimentary layers are anisotropic media. Time-lapse seismic monitoring is crucial for many applications, such as geologic carbon utilization and storage (including potential $\mathrm{CO}_{2}$ leakage through fracture/fault zones), geothermal energy production, conventional/unconventional oil and gas production, waste water injection, and waste repository, etc. Therefore, the optimal design of time-lapse seismic surveys is essential for reliable and cost-effective monitoring of subsurface changes.

Conventional time-lapse seismic surveys are usually based on seismic wavefield illumination $[5,11]$, employing ray-based methods $[2,13]$ and wave-equation based methods $[1,25]$. However, complex geology limits the practical applications of these methods. [6] designed optimal time-lapse seismic surveys based on elastic-wave sensitivity modeling, that is, numerically propagating the sensitivity of an elastic wavefield with respect to medium parameters such as P-wave velocity $V_{P}$ or mass density $\rho$. This approach has a significant advantage that the spatial distributions of the elastic-wave sensitivity energies indicates how much significant information of the time-lapse changes of reservoirs can be acquired using a given geophone distribution. This approach has been applied to monitoring $\mathrm{CO}_{2}$ leakage through faults [21]. However, present elastic-wave sensitivity methods are limited to isotropic media, which can be simply described by the density $\rho$, P-wave velocity $V_{P}$, S-wave velocity $V_{S}$, and certain derived petrophysical parameters such as the ratio of fluid saturation. However, additional parameters are needed to characterize anisotropic properties in fracture/fault zones such as elasticity parameters $c_{i j k l}$, excess compliance $\Delta s_{i j k l}$ contributed from fractures $[8,17]$ and Thomsen parameters along with a reference velocity $[23,24]$. 
We develop a novel method for numerical modeling of anisotropic elastic-wave sensitivity propagation for optimal design of cost-effective time-lapse seismic surveys. In this method, we directly simulate propagation of elastic-wave sensitivity wavefields resulting from time-lapse changes in target monitoring regions using the same elastic-wave equation with a secondary source term. We study how subsurface anisotropic media including the fracture anisotropy induced by fracture alignments, affects the detection of time-lapse changes of anisotropic media. Our method follows the similar strategy used in the elastic-wave sensitivity propagation in isotropic media [6]. First, we separate the $\mathrm{qP}-$ and $\mathrm{qS}$-waves from the total elastic wavefield. Second, we introduce criteria for monitoring anisotropic fracture zones based on the sensitivities and energies of the $\mathrm{qP}$ - and qS-waves. Third, to adequately model the anisotropy, we simulate the propagation of anisotropic elastic-wave sensitivity with respect to $c_{i j k l}$ and $\rho$, instead of $V_{P}, V_{S}$ and $\rho$ as in [6]. When anisotropy parameters vary, the sensitivity wavefields vary, and in consequence the monitoring criteria alter their values. We verify this hypothesis using the following numerical tests. First, we construct an anisotropic model modified from the Hess vertical transverse isotropic (VTI) benchmark model, where different types of anisotropy exist in the target fracture zone, in addition to anisotropic sedimentary layers. Receiver lines locate either horizontally or in vertical wells for time-lapse surface seismic monitoring or time-lapse vertical seismic profiling monitoring. Second, we compute the spatial distributions of the $\mathrm{qP}$ - and $\mathrm{qS}$-waves sensitivity energies. Third, we study how the fracture anisotropy can affect the spatial distributions of anisotropic elastic-wave sensitivity energies.

We organize the paper as follows. In the Methodology section, we present the theory of anisotropic elastic-wave sensitivity propagation, and describe the criteria for monitoring time-lapse changes in anisotropic media. In the section of Numerical Results, we conduct a series of numerical tests involving a modified Hess VTI model, to analyze how the anisotropic characteristics of fracture zones influence the elastic-wave sensitivity propagation. We summarize our findings in the Conclusions section.

\section{Methodology}

The elastic-wave equation in anisotropic media in the stress-velocity form is given by [3]:

$$
\begin{aligned}
& \frac{\partial \sigma_{i j}}{\partial t}=\frac{1}{2} c_{i j k l}\left(\frac{\partial v_{k}}{\partial x_{l}}+\frac{\partial v_{l}}{\partial x_{k}}\right), \\
& \frac{\partial v_{i}}{\partial t}=\frac{1}{\rho} \frac{\partial \sigma_{i j}}{\partial x_{j}}+f_{i},
\end{aligned}
$$

where $\sigma_{i j}=\sigma_{i j}(\mathbf{x}, t)$ is the stress tensor, $v_{i}=v_{i}(\mathbf{x}, t)$ is the particle velocity vector, $c_{i j k l}=c_{i j k l}(\mathbf{x})$ is the fourth-order elasticity tensor, $\rho$ is the mass density. The variable $\mathbf{x}$ represents spatial coordinates. Besides, $f_{i}=f_{i}\left(\mathbf{x}_{s}, t\right) \delta\left(\mathbf{x}_{s}\right)$ is the external force vector adding to the particle 
velocity wavefield. This external force could also be added to the stress. In the following, we ignore the external force term without loss of generality.

The concept of elastic sensitivity propagation was introduced in $[6,21]$ to study elasticwavefield changes caused by the variation of a certain medium parameter in target monitoring regions during time-lapse seismic monitoring. In contrast to the conventional data analysis approach where the seismic data are simply compared using the waveform difference, this approach computes the partial derivative of the elastic wavefield with respect to the investigated medium parameter, and simulates the propagation of this quantity using the same formulation as the elastic-wave equation but with a different source term. The accumulated energy of this elastic-wave sensitivity in time and space can be used as an indicator of how strongly the elastic wavefield is affected by the change of the investigated medium parameter, and therefore, facilitates cost-effective time-lapse seismic survey design.

Specifically, the sensitivity of elastic wavefield with respect to a certain medium property parameter $\xi$ (the components of $c_{i j k l}$ and the mass density $\rho$ for a linear elastic medium) is defined as $\partial \phi / \partial \xi$, where $\phi=(\sigma, \mathbf{v})$ is the elastic wavefield. By taking the derivative of Eq. (2.1) with respect to $\xi$, we obtain the following elastic-wave sensitivity equations [6,21], with some modifications:

$$
\begin{aligned}
\frac{\partial}{\partial t}\left(\frac{\partial \sigma_{i j}}{\partial \xi}\right) & =\frac{1}{2} c_{i j k l}\left[\frac{\partial}{\partial x_{l}}\left(\frac{\partial v_{k}}{\partial \xi}\right)+\frac{\partial}{\partial x_{k}}\left(\frac{\partial v_{l}}{\partial \xi}\right)\right]+\frac{1}{2} \frac{\partial c_{i j k l}}{\partial \xi}\left(\frac{\partial v_{k}}{\partial x_{l}}+\frac{\partial v_{l}}{\partial x_{k}}\right) \delta\left(\mathbf{x}_{\text {target }}\right), \\
\frac{\partial}{\partial t}\left(\frac{\partial v_{i}}{\partial \xi}\right) & =\frac{1}{\rho} \frac{\partial}{\partial x_{j}}\left(\frac{\partial \sigma_{i j}}{\partial \xi}\right)-\frac{1}{\rho^{2}} \frac{\partial \rho}{\partial \xi} \frac{\partial \sigma_{i j}}{\partial x_{j}} \delta\left(\mathbf{x}_{\text {target }}\right),
\end{aligned}
$$

where $\mathbf{x}_{\text {target }}$ indicates the location of target monitoring region(s). It can be either a target monitoring region or distinctive monitoring regions when applying our method to design of time-lapse seismic surveys. Therefore, the meaning of $\delta\left(\mathbf{x}_{\text {target }}\right)$ can be interpreted as a spatial mask where the sensitivity source terms are applicable, and only when the elastic wavefield touches the target monitoring region(s), the elastic-wave sensitivity wavefield is generated.

Rather than using the elastic-wave sensitivities with respect to $\mathrm{P}-$, $\mathrm{S}$-wave velocities or mass density $\rho$ as in the isotropic case, we study the elastic-wave sensitivities with respect to elasticity parameters $c_{i j k l}$ and $\rho$ in anisotropic media. These elastic parameters no longer have simple relationships with the wave velocities in anisotropic media, which vary along different propagation directions. Using the Voigt notation [3], the fourth-order $c_{i j k l}$ can be converted to an elasticity matrix $C_{I J}$ where $I, J=1,2, \cdots, 6$.

In this paper, we focus on the $2 \mathrm{D}$ case and assume that the coordinate plane is the $x_{1} x_{3}$ coordinate plane. Therefore, $I, J=1,3,5$, and the elastic-wave sensitivity propagation equations with respect to the elasticity parameter $C_{I J}$, e.g., $C_{11}$, are explicitly written as

$$
\frac{\partial \Xi_{11}^{C_{11}}}{\partial t}=C_{11} \frac{\partial W_{1}^{C_{11}}}{\partial x_{1}}+C_{13} \frac{\partial W_{3}^{C_{11}}}{\partial x_{3}}+C_{15}\left(\frac{\partial W_{3}^{C_{11}}}{\partial x_{1}}+\frac{\partial W_{1}^{C_{11}}}{\partial x_{3}}\right)+\frac{\partial v_{1}}{\partial x_{1}} \delta\left(\mathbf{x}_{\text {target }}\right),
$$




$$
\begin{aligned}
& \frac{\partial \Xi_{33}^{C_{11}}}{\partial t}=C_{13} \frac{\partial W_{1}^{C_{11}}}{\partial x_{1}}+C_{33} \frac{\partial W_{3}^{C_{11}}}{\partial x_{3}}+C_{35}\left(\frac{\partial W_{3}^{C_{11}}}{\partial x_{1}}+\frac{\partial W_{1}^{C_{11}}}{\partial x_{3}}\right), \\
& \frac{\partial \Xi_{13}^{C_{11}}}{\partial t}=C_{15} \frac{\partial W_{1}^{C_{11}}}{\partial x_{1}}+C_{35} \frac{\partial W_{3}^{C_{11}}}{\partial x_{3}}+C_{55}\left(\frac{\partial W_{3}^{C_{11}}}{\partial x_{1}}+\frac{\partial W_{1}^{C_{11}}}{\partial x_{3}}\right), \\
& \frac{\partial W_{1}^{C_{11}}}{\partial t}=\frac{1}{\rho}\left(\frac{\partial \Xi_{11}^{C_{11}}}{\partial x_{1}}+\frac{\partial \Xi_{11}^{C_{13}}}{\partial x_{3}}\right), \\
& \frac{\partial W_{3}^{C_{11}}}{\partial t}=\frac{1}{\rho}\left(\frac{\partial \Xi_{13}^{C_{11}}}{\partial x_{1}}+\frac{\partial \Xi_{33}^{C_{11}}}{\partial x_{3}}\right),
\end{aligned}
$$

in which the elastic-wave sensitivity wavefields are

$$
\Xi_{11}^{C_{11}}=\frac{\partial \sigma_{11}}{\partial C_{11}}, \quad \Xi_{33}^{C_{11}}=\frac{\partial \sigma_{33}}{\partial C_{11}}, \quad \Xi_{13}^{C_{11}}=\frac{\partial \sigma_{13}}{\partial C_{11}}, \quad W_{1}^{C_{11}}=\frac{\partial v_{1}}{\partial C_{11}}, \quad W_{3}^{C_{11}}=\frac{\partial v_{3}}{\partial C_{11}} .
$$

The elastic-wave sensitivity wavefield with respect to the other $C_{I J}$ parameters and density $\rho$ can be derived analogously. The above formulation can only simulate $\mathrm{qP}$ - and qSV-waves in 2D space. For 3D applications in anisotropic media, the corresponding $3 \mathrm{D}$ elastic-wave sensitivity equation can be derived analogously, where all the wavefield components, including qP-, qSV- and qSH-waves can be properly simulated.

For generally anisotropic media where $C_{15}$ and $C_{35}$ might be nonzero, $C_{11}$ is generally not equal to $C_{33}$, and $C_{13}$ might not be equal to $C_{11}-2 C_{55}$ as that in isotropic media. The complexity of these elastic parameters provides a greater flexibility to accurately describe the anisotropy of fractured reservoirs compared to the isotropic case.

Effective medium theories [18] show that the values of elasticity parameters can be influenced by the spatial alignment, shape, aspect ratio and density of the fractures. Therefore, elastic-wave sensitivity may also be influenced with these aforementioned petrophysical parameters. In this paper, we only study the elastic-wave sensitivity with respect to elasticity parameters $C_{I J}$.

Multicomponent seismic data acquisition and subsequent data processing flow usually separate the P- and S-wave components from seismic data to facilitate relevant geological and petrophysical interpretations. Therefore, we should set the monitoring objective functions based on separated P- and S-wave sensitivities with respect to the medium parameter $\xi[6,21]$.

In anisotropic media, the elastic wavefield consists of qP- and qS-wavefields instead of pure $\mathrm{P}$ - and S-wavefields. We define the time-lapse monitoring criteria based on the energies of decomposed $\mathrm{qP}$ - and qS-wave sensitivity displacement wavefield as

$$
E_{q P}^{\xi}(\mathbf{x})=\int_{0}^{T}\left|\mathbf{u}_{q P}^{\xi}(\mathbf{x}, t)\right|^{2} d t, \quad E_{q S}^{\xi}(\mathbf{x})=\int_{0}^{T}\left|\mathbf{u}_{q S}^{\xi}(\mathbf{x}, t)\right|^{2} d t,
$$

where $T$ is the total time duration of wavefield propagation. Because the elastic-wave equation (2.1) is based on stress and particle velocity wavefield propagation, we obtain 
the displacement sensitivity wavefield by integrating the particle velocity wavefield in time, i.e.,

$$
\mathbf{u}^{\xi}(\mathbf{x}, t)=\left[u_{1}^{\xi}(\mathbf{x}, t), u_{3}^{\xi}(\mathbf{x}, t)\right]=\left[\int_{0}^{t} W_{1}^{\xi}(\mathbf{x}, \tau) d \tau, \int_{0}^{t} W_{3}^{\xi}(\mathbf{x}, \tau) d \tau\right] .
$$

Conventional time-lapse analyses usually use a portion (a given time window and a number of traces) of the wavefield interacting with the target monitoring regions. In comparison, our energy criteria in Eq. (2.5) account for the accumulated spatial distribution of the energy of elastic-wavefield sensitivities resulting from time-lapse changes in target monitoring regions. This characteristics provides comprehensive information regarding influences of time-lapse changes, such as the spatial paths and distributions of the wavefield energy.

To compute the energy criteria, we must decompose qP- and qS-sensitivity wavefields from the total elastic-wave sensitivity wavefield. However, this decomposition is nontrivial in general anisotropic media, because the wave mode separation cannot be simply achieved using such as the Helmholtz decomposition as in isotropic media [6, 21]. We decompose the vector $\mathrm{qP}$ - and $\mathrm{qS}$-wave sensitivity wavefield from the total displacement sensitivity wavefield as

$$
\mathbf{u}_{q P}^{\xi}(\mathbf{x}, t)=\mathcal{L}_{q P}\left[\mathbf{u}^{\xi}(\mathbf{x}, t)\right], \quad \mathbf{u}_{q S}^{\xi}(\mathbf{x}, t)=\mathcal{L}_{q S}\left[\mathbf{u}^{\xi}(\mathbf{x}, t)\right],
$$

and $\mathbf{u}^{\tilde{\xi}}(t)$ is the total elastic-wave sensitivity wavefield with respect to medium parameter $\xi$, and $\mathcal{L}$ is a wavefield separator operating on the total elastic-wave sensitivity wavefield to obtain the vector $\mathrm{qP}$ - and $\mathrm{qS}$-sensitivity wavefields. We adopt the low-rank decomposition scheme developed in [4] to achieve accurate decomposition in complex anisotropic media. We apply the low-rank decomposition to the total elastic-wave sensitivity wavefield at each time step during wavefield modeling.

For complex anisotropic media, the CPU time consumption of this decomposition can be even higher than that used for wavefield modeling. We therefore employ a hybrid time- and frequency-domain modeling approach to elastic-wave sensitivity modeling to obtain the monitoring criteria. We compute the frequency-domain sensitivity wavefield during sensitivity wavefield propagation using a discrete Fourier transform [22]:

$$
\mathbf{U}^{\tilde{\xi}}(\mathbf{x}, \omega)=\sum_{t=0}^{T} \mathbf{u}^{\xi}(\mathbf{x}, t) e^{-i \omega t},
$$

where $\omega$ is the angular frequency, $T$ is the maximum wavefield propagation time, and $\mathbf{U}^{\mathcal{F}}(\mathbf{x}, \omega)$ is the frequency-domain sensitivity wavefield. We conduct this integration operation during the time-domain modeling. This operation is essentially a grid-wise operation, i.e., Eq. (2.8) is computed for each spatial grid point independently. Therefore, the operation can be simply parallelized and consumes trivial additional CPU time.

Because the low-rank decomposition is a purely spatial operation, the $\mathrm{qP}$ - and $\mathrm{qS}$ sensitivity wavefields in the frequency domain are

$$
\mathbf{U}_{q P}^{\xi}(\mathbf{x}, \omega)=\mathcal{L}_{q P}\left[\mathbf{U}^{\xi}(\mathbf{x}, \omega)\right], \quad \mathbf{U}_{q S}^{\xi}(\mathbf{x}, \omega)=\mathcal{L}_{q S}\left[\mathbf{U}^{\xi}(\mathbf{x}, \omega)\right],
$$


which leads to

$$
E_{q P}^{\xi}(\mathbf{x})=\int_{\omega_{\min }}^{\omega_{\max }}\left|\mathbf{U}_{q P}^{\xi}(\mathbf{x}, \omega)\right|^{2} d \omega, \quad E_{q S}^{\xi}(\mathbf{x})=\int_{\omega_{\min }}^{\omega_{\max }}\left|\mathbf{U}_{q S}^{\xi}(\mathbf{x}, \omega)\right|^{2} d \omega,
$$

where $\left|\mathbf{U}^{\tilde{\xi}}(\mathbf{x}, \omega)\right|^{2}=\Re\left[\mathbf{U}^{\tilde{\xi}}(\mathbf{x}, \omega)\right]^{2}+\Im\left[\mathbf{U}^{\tilde{\xi}}(\mathbf{x}, \omega)\right]^{2}$, and $\omega_{\min }$ and $\omega_{\max }$ are the minimum and maximum frequency of interest, respectively.

Eq. (2.10) indicates that we only need to conduct the qP-qS decomposition for a few selected frequencies, instead of for each time step in the time-domain modeling approach, and can obtain the band-limited approximate monitoring criteria to those in Eq. (2.5). With appropriate choices of $\omega_{\min }, \omega_{\max }$ and the frequency sampling interval $\Delta \omega$, the Nyquist-Shannon sampling theorem tells us that the time-domain monitoring criteria can be accurately reconstructed. Therefore, the frequency-domain monitoring criteria in Eq. (2.10) can serve as a computationally cost-effective monitoring criteria. The computational cost of this hybrid time- and frequency-domain modeling approach is therefore significantly reduced compared to that based on the time-domain modeling approach for anisotropic media. In the following numerical example, we find that the computational time cost using the hybrid time- and frequency-domain approach is only approximately 10 percent of that of the pure time-domain approach.

\section{Numerical results}

The Hess Corporation built a 2D VTI model for testing finite-difference modeling of anisotropic wave propagation. This model consists of transversely isotropic media with a vertical symmetry axis, or VTI media. The original Hess model contains a fairly low qS-wave velocity. We use a modified Hess model to verify our anisotropic elastic-wave sensitivity modeling method. We modify the grid size of the original Hess model and the qS-wave velocities to make it suitable for our numerical modeling of the elastic-wave sensitivity propagation.

The modified Hess model includes a set of elasticity parameter $C_{I J}$ models and a density model, as shown in Figs. 1a-e. The model is VTI anisotropic throughout the space except in the high-velocity salt body in the left half of the model indicated by the red color region in Figs. 1a-d. To quantitatively compare the influences of background anisotropy properties on the spatial distribution of sensitivity energies, we also create an isotropic Hess model using $C_{33}=C_{11}$ and $C_{13}=C_{11}-2 C_{55}$. The dimensions of the modified model are $4.8 \mathrm{~km}$ in the horizontal direction and $2 \mathrm{~km}$ in the vertical direction. We discretize the modified model using 601 grids in the horizontal direction and 251 grids in the vertical directions, with a grid interval of $8 \mathrm{~m}$ in both directions. For comparison, the grid size of the original Hess VTI model is $20 \mathrm{ft}$, i.e., approximately $6.1 \mathrm{~m}$, in both spatial directions. The original Hess VTI model consists of 1501 grid points in the depth direction, and 3617 grid points in the horizontal direction. Therefore, we roughly shrink the original model by 4.6 times in both directions. 


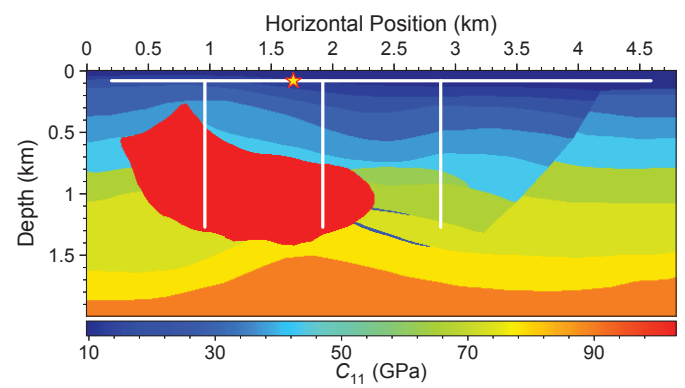

(a) $C_{11}$ model

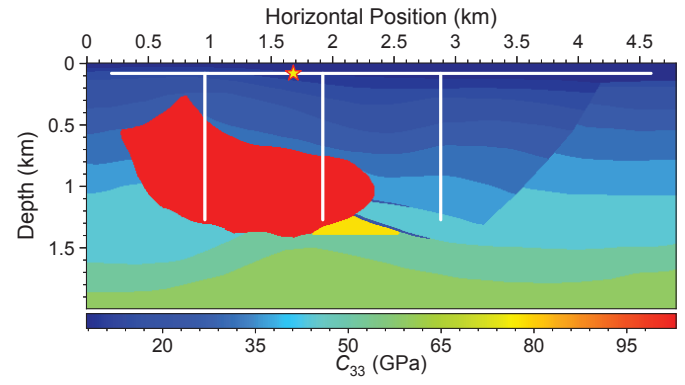

(c) $C_{33}$ model

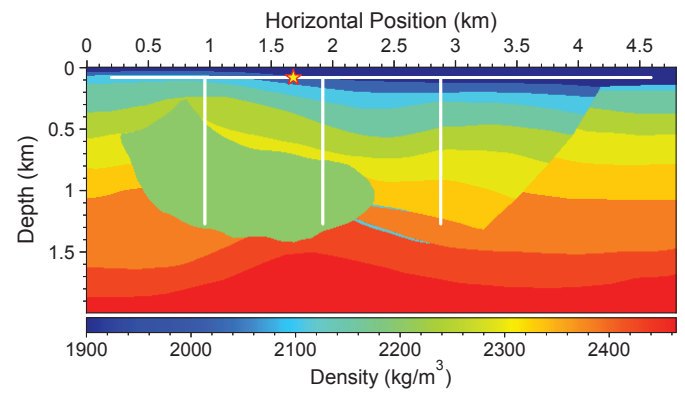

(e) Density model

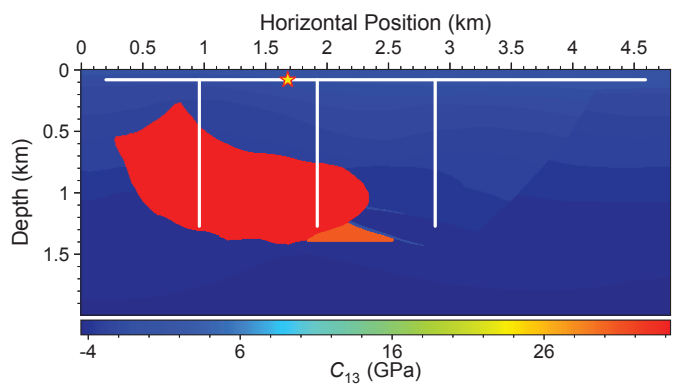

(b) $C_{13}$ model

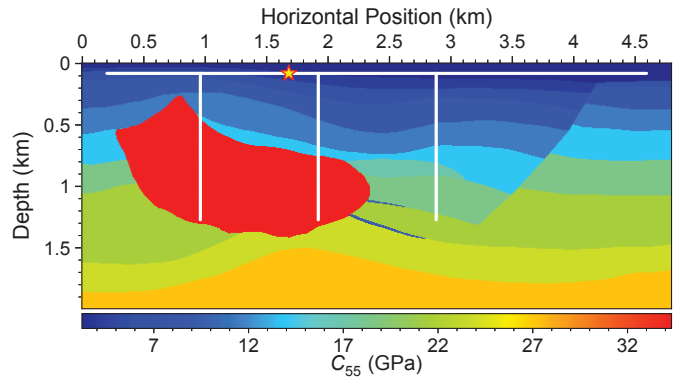

(d) $C_{55}$ model

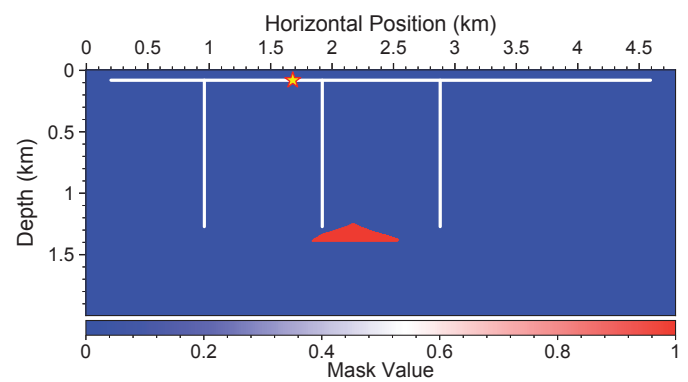

(f) Mask model

Figure 1: The modified Hess anisotropic model: (a)-(d) show the elasticity parameters, and (e) displays the density. The target monitoring fracture zone is shown in ( $f)$. The yellow star indicates the source position, and the white lines refer to the positions of surface and vertical-well receivers.

We set a target monitoring fracture zone indicated by the red-color regions in Fig. 1f. We assume the monitoring fracture zone to be isotropic (ISO) or transversely isotropic with a tilted axis (TTI). Effective medium theories show that the isotropy may result from randomly distributed fractures with random orientations. The TTI can result from a set of fractures with tilted fracture surface normal. We assume the tilted angle of this TTI fracture zone is 45 degrees with respect to the vertical axis. Therefore, we conduct a total of four sensitivity modeling tests by combining the ISO or the VTI background with the ISO or the TTI target monitoring zone. 
We perform numerical modeling of elastic-wave sensitivity propagation with an explosive source at $1,680 \mathrm{~m}$ along the horizontal direction with a depth of $80 \mathrm{~m}$. The temporal signature of the sources is a Ricker wavelet with a center frequency of $15 \mathrm{~Hz}$. We indicate the source locations using yellow stars in Figs. 1a-e. For surface seismic monitoring, we use a total of 550 receivers located from $200 \mathrm{~m}$ to $4,592 \mathrm{~m}$ in the horizontal direction with a depth of $80 \mathrm{~m}$. For vertical seismic profiling (VSP) monitoring, we use three vertical receiver wells to receive the elastic-wave sensitivity. The receiver interval is $8 \mathrm{~m}$ for both surface seismic surveys and VSP surveys. We denote these surface and VSP receivers using the white horizontal and vertical lines in Fig. 1.

We conduct modeling of the anisotropic elastic-wave sensitivity propagation using a high-order rotated staggered-grid finite-difference method [7]. The total length of wavefield propagation is $4 \mathrm{~s}$. We then compute the spatial distributions of anisotropic elasticwave sensitivity energies using Eq. (2.10) for qP- and qS-wave sensitivities with respect to elasticity parameters $C_{11}$ and $C_{55}$.

To demonstrate the concept of elastic-wave sensitivity, we show the snapshots of the elastic wavefield and the elastic-wave sensitivity wavefield in Fig. 2. Figs. 2a, c and e show the snapshots at $0.4,0.6$ and $0.8 \mathrm{~s}$, respectively, of the $x_{3}$-component of the elastic displacement wavefield. Once the elastic wavefield touches the target monitoring zone, the elastic-wave sensitivity wavefield is generated, as shown in Figs. $2 b, d$ and f. As time evolves, the elastic-wave sensitivity wavefield propagates through the space and arrives at geophones. In this example, we assume the background is VTI and the fracture zone is TTI, and the wavefield is stimulated for the elasticity parameter $C_{11}$. For different medium properties or target monitoring parameters, the resulting sensitivity wavefields are generally different, following the elastic-wave sensitivity propagation equation (2.3).

We show the spatial distributions of $\mathrm{qP}$ - and qS-wave sensitivity energies with respect to the elasticity parameters $C_{11}$ and $C_{55}$ in Figs. 3 and 4, respectively. These quantities are computed using the energy criteria in Eq. (2.10). They are computed by integrating the energies of the $\mathrm{qP}$ - and $\mathrm{qS}$-sensitivity wavefields over time.

Figs. 3a-d show the $\mathrm{qP}$-wave sensitivity energies with respect to the elasticity parameter $C_{11}$ where the fracture zone is ISO or TTI, and the background is ISO or VTI. The spatial distributions of $E_{q P}^{C_{11}}$ between the cases of ISO and TTI fracture zones are very similar in respective ISO and VTI backgrounds. However, different backgrounds obviously lead to different energy concentrations in space for the same type of fracture zone. For instance, in the ISO background, the TTI fracture zone leads to a wide energy concentration at the surface from about 3 to $4.6 \mathrm{~km}$, while the concentration is notably narrower in the VTI background.

Figs. 3e-h show the qS-wave sensitivity energies with respect to the elasticity parameter $C_{11}$ where the fracture zone is ISO or TTI, and the background is ISO or VTI. Similar to the $\mathrm{qP}$-wave sensitivity case, the spatial distributions of $E_{q S}^{C_{11}}$ between the cases of ISO and TTI fracture zones are similar to each other in respective ISO and VTI backgrounds, yet different backgrounds result in different energy concentrations in space for the same 


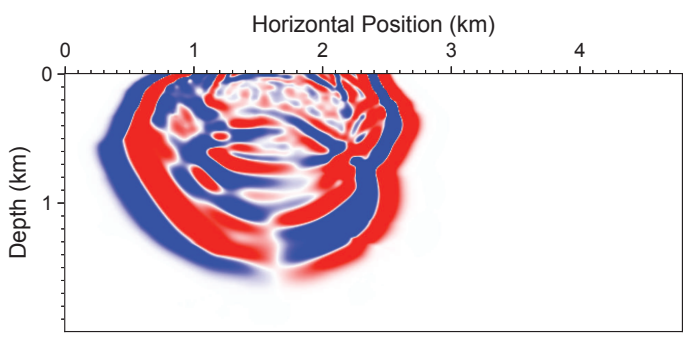

(a) $t=0.4 \mathrm{~s}$

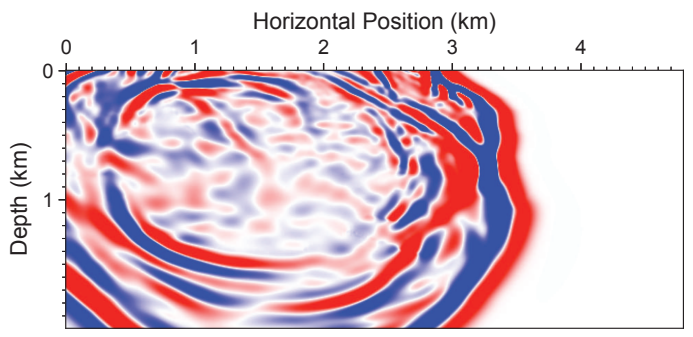

(c) $t=0.6 \mathrm{~s}$

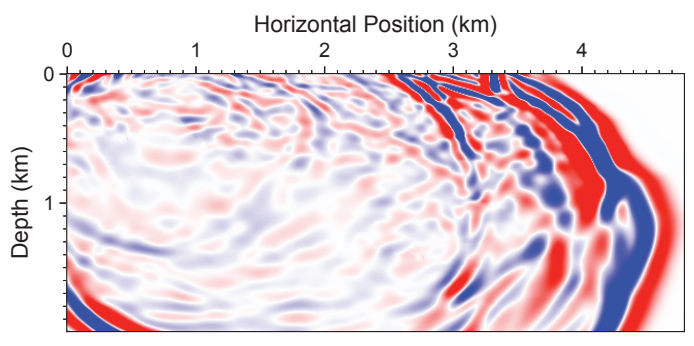

(e) $t=0.8 \mathrm{~s}$

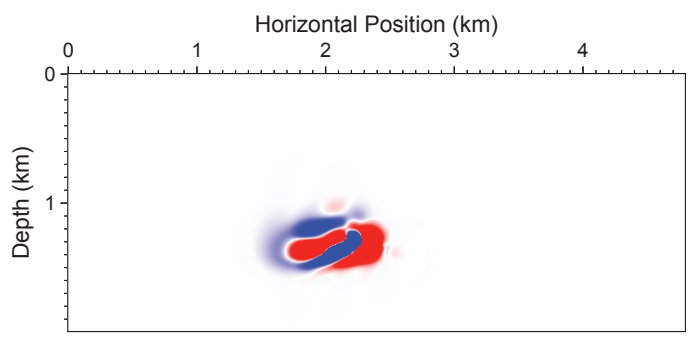

(b) $t=0.4 \mathrm{~s}$

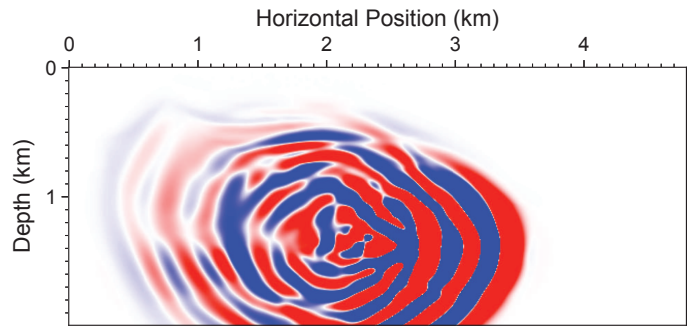

(d) $t=0.6 \mathrm{~s}$

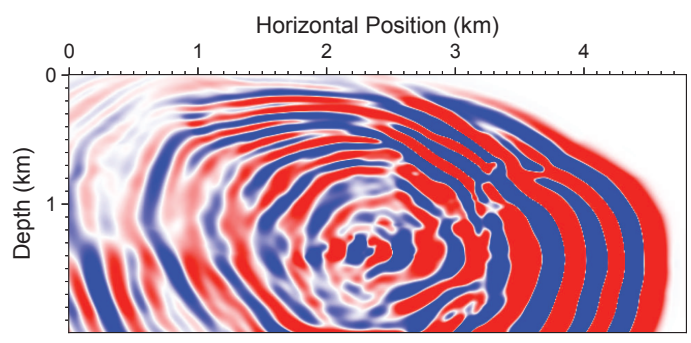

(f) $t=0.8 \mathrm{~s}$

Figure 2: Wavefield snapshots of the $x_{3}$-component of the elastic displacement wavefield $(\mathrm{a}, \mathrm{c}, \mathrm{e})$ and the $x_{3}$-component of the elastic-wave sensitivity wavefield $(\mathrm{b}, \mathrm{d}, \mathrm{f})$. The snapshot time for $(\mathrm{a})$ and $(\mathrm{b})$ is $0.4 \mathrm{~s}$, $0.6 \mathrm{~s}$ for $(\mathrm{c})$ and $(\mathrm{d})$, and $0.8 \mathrm{~s}$ for $(\mathrm{e})$ and $(\mathrm{f})$. The background is VTI and the fracture zone is TTI. These wavefields are associated with the change of $C_{11}$.

type of fracture zone. In this case, however, the qS-wave sensitivity energy distribution at the surface for the ISO fracture zone in the VTI background, which is approximately from 3.2 to $3.9 \mathrm{~km}$, is slightly narrower than that with the TTI fracture zone, which is approximately from 3.0 to $4.3 \mathrm{~km}$.

The surface distributions of $E_{q P}^{C_{11}}$ and $E_{q S}^{C_{11}}$ are shown in Figs. $5 \mathrm{a}$ and b, respectively, for the aforementioned four tests. Specially, we find that in the VTI background model, both the ISO and TTI fracture zones lead to two notable energy concentrations, while the ISO background model leads to only one notable concentration. More specifically, for the VTI background model, the locations of the maximum energy distribution for the ISO fracture zone and the TTI fracture zone are exchanged with their second maximum energy 


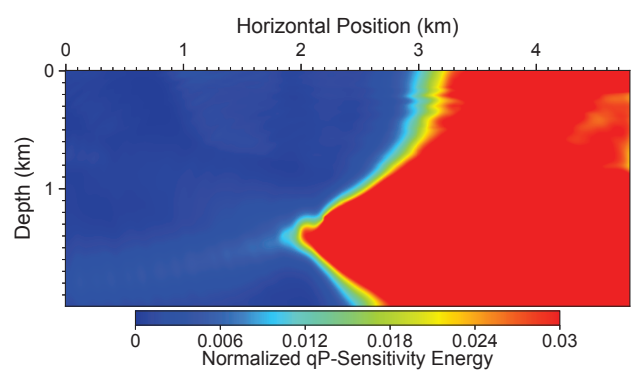

(a) $E_{q P}^{C_{11}}$ with ISO fracture and ISO background

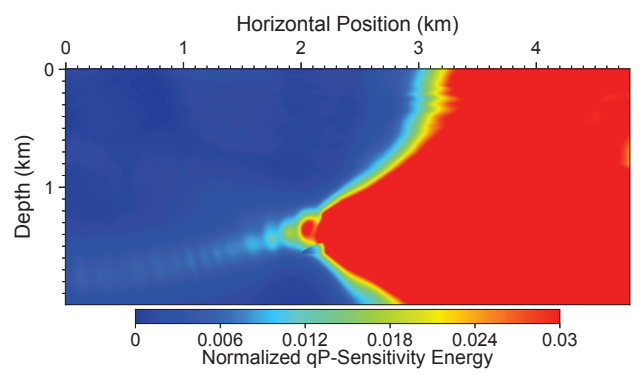

(c) $E_{q P}^{C_{11}}$ with TTI fracture and ISO background

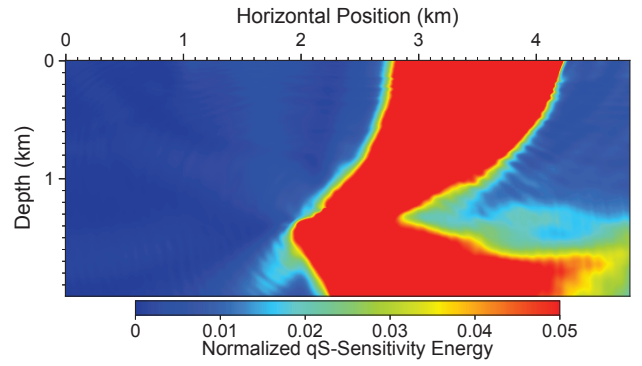

(e) $E_{q S}^{C_{11}}$ with ISO fracture and ISO background

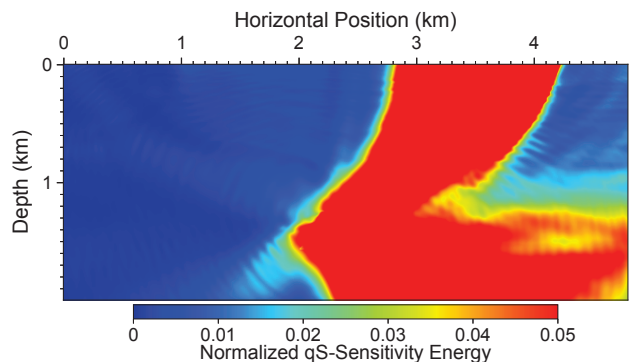

(g) $E_{q S}^{C_{11}}$ with TTI fracture and ISO background

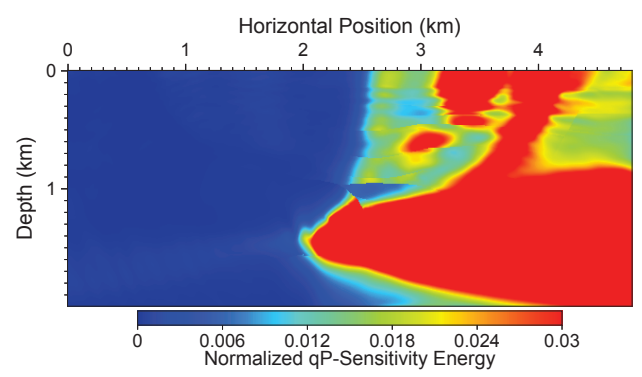

(b) $E_{q P}^{C_{11}}$ with ISO fracture and VTI background

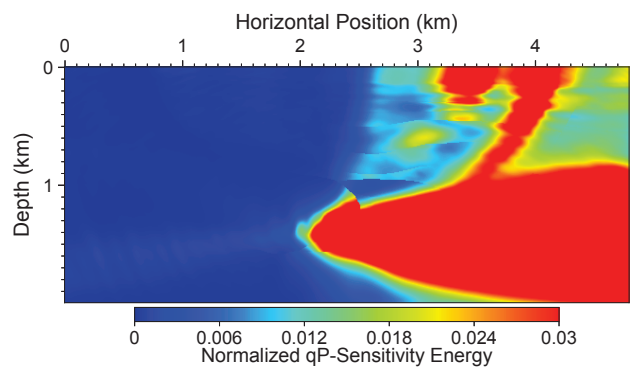

(d) $E_{q P}^{C_{11}}$ with TTI fracture and VTI background

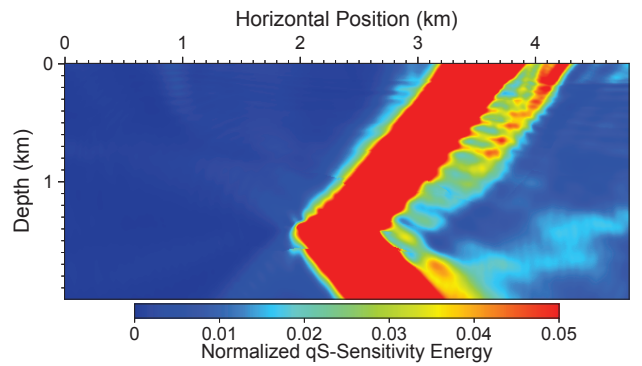

(f) $E_{q S}^{C_{11}}$ with ISO fracture and VTI background

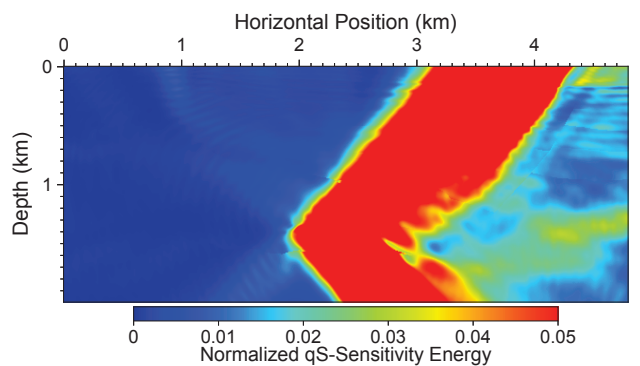

(h) $E_{q S}^{C_{11}}$ with TTI fracture and VTI background

Figure 3: Spatial distributions of $q \mathrm{P}-(\mathrm{a}, \mathrm{b}, \mathrm{c}, \mathrm{d})$ and $\mathrm{qS}$-wave $(\mathrm{e}, \mathrm{f}, \mathrm{g}, \mathrm{h})$ sensitivity energies with respect to the elasticity parameter $C_{11}$ for the ISO fracture zone $(a, b, e, f)$ and the TTI fracture zone $(c, d, g, h)$, with an ISO background (a, c, e, g) and a VTI background (b, d, f, h). 


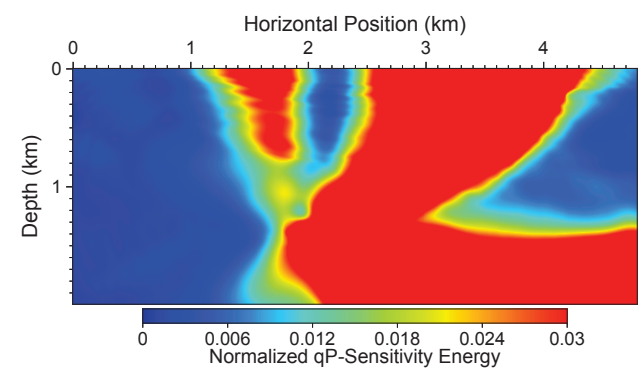

(a) $E_{q P}^{C_{55}}$ with ISO fracture and ISO background

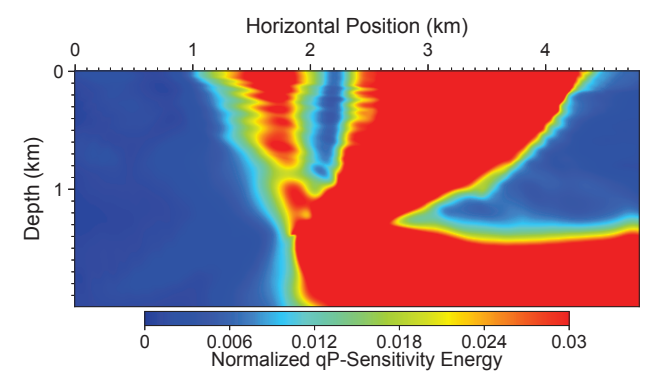

(c) $E_{q P}^{C_{55}}$ with TTI fracture and ISO background

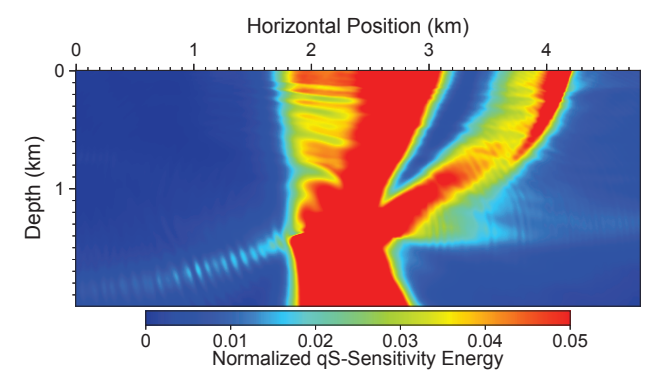

(e) $E_{q S}^{C_{55}}$ with ISO fracture and ISO background

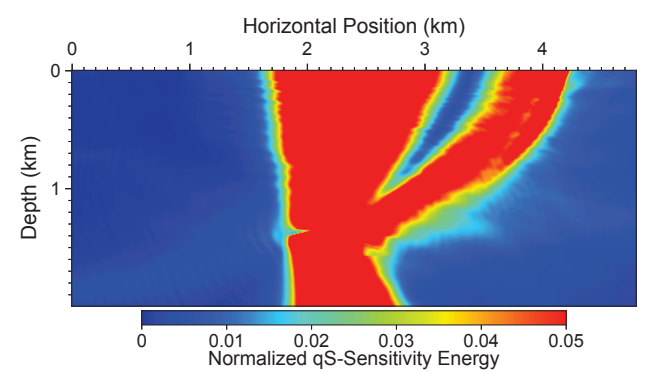

(g) $E_{q S}^{C_{55}}$ with TTI fracture and ISO background

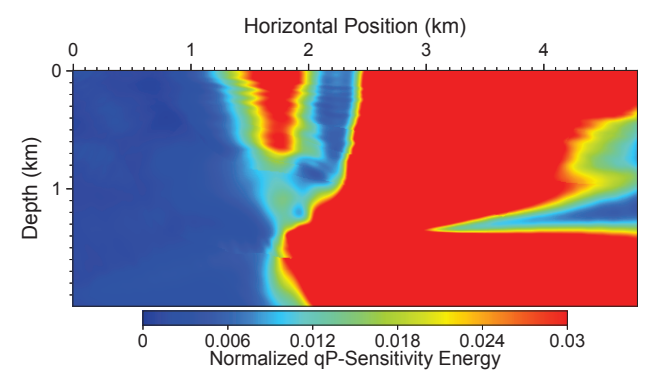

(b) $E_{q P}^{C_{55}}$ with ISO fracture and VTI background

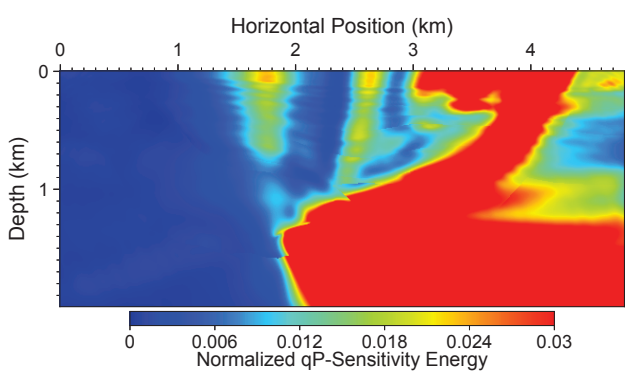

(d) $E_{q P}^{C_{55}}$ with TTI fracture and VTI background

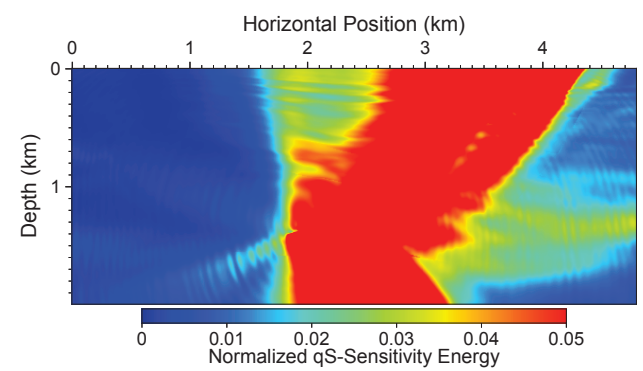

(f) $E_{q S}^{C_{55}}$ with ISO fracture and VTI background

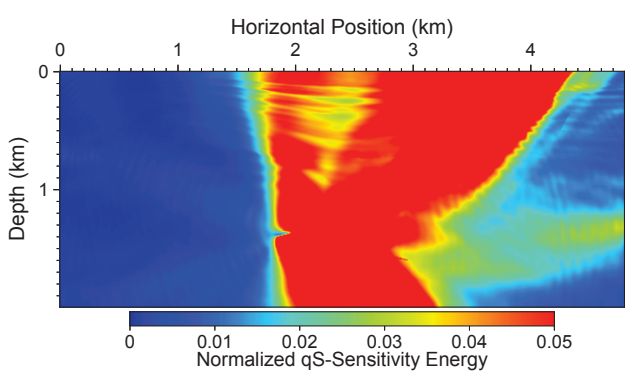

(h) $E_{q S}^{C_{55}}$ with TTI fracture and VTI background

Figure 4: Spatial distributions of $\mathrm{qP}-(\mathrm{a}, \mathrm{b}, \mathrm{c}, \mathrm{d})$ and $\mathrm{qS}$-wave $(e, f, g, h)$ sensitivity energies with respect to the elasticity parameter $C_{55}$ for the ISO fracture zone $(a, b, e, f)$ and the TTI fracture zone $(c, d, g, h)$, with an ISO background (a, c, e, g) and a VTI background (b, d, f, h). 


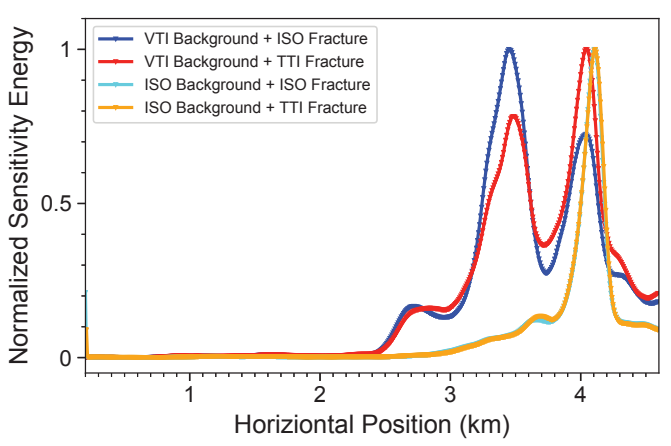

(a) $E_{q P}^{C_{11}}$

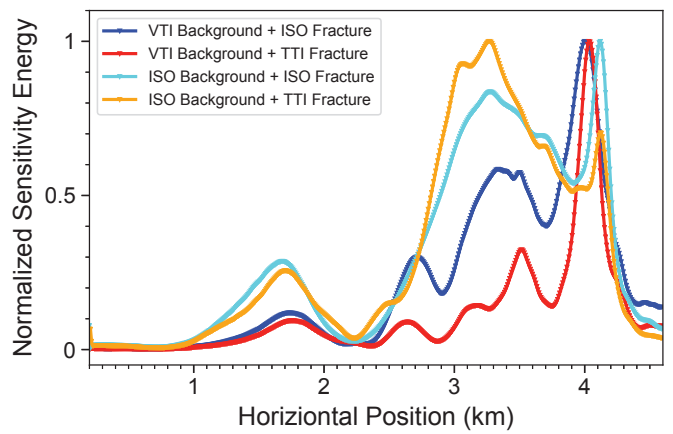

(c) $E_{q P}^{C_{55}}$

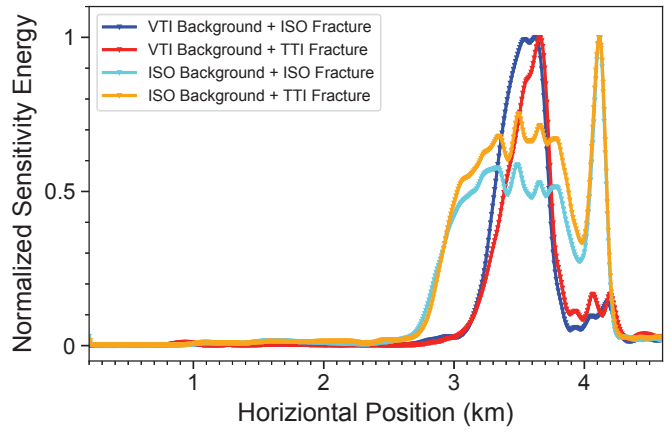

(b) $E_{q S}^{C_{11}}$

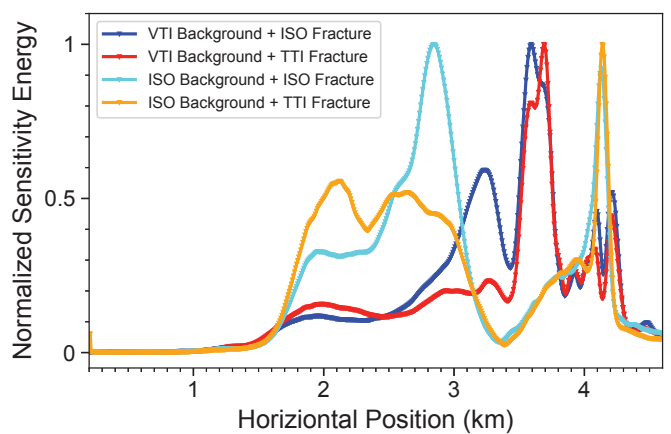

(d) $E_{q S}^{C_{55}}$

Figure 5: The normalized $\mathrm{qP}$-wave sensitivity energies with respect to the elasticity parameters $C_{11}$ in $(\mathrm{a})$ and $C_{55}$ in (c), and the normalized qS-wave sensitivity energies with respect to the elasticity parameters $C_{11}$ in (b) and $C_{55}$ in (d), for different fracture zone properties and backgrounds.

concentration locations. This result shows that different fracture zone properties in the same background model lead to different elastic-wave-sensitivity-energy concentrations.

For the $E_{q S}^{C_{11}}$ distribution, we find that an anisotropic background leads to a different energy concentration compared with that in an isotropic background model, and the difference between the maximum energy concentration locations can be as large as $1 \mathrm{~km}$ in the horizontal direction.

We show the qP- and qS-wave sensitivity energies associated with the elasticity parameter $C_{55}$ in Fig. 4. The comparisons on the energy's spatial distributions among the four numerical tests resemble to those for the elasticity parameter $C_{11}$. In this case, however, the sensitivity energy's surface concentration is slightly more complicated than that with respect to $C_{11}$, as shown in Figs. $5 c$ and $d$ for $\mathrm{qP}$ - and qS-wave sensitivity energies, respectively.

We find that the $\mathrm{qP}$-wave sensitivity distribution for the TTI fracture zone and the VTI background model is most concentrated at approximately $4 \mathrm{~km}$, while the other three cases have obviously wider distribution at the surface. Specifically, the energy distri- 
bution associated the TTI fracture zone in the ISO background model has a completely different maximum energy concentration location at approximately $3.2 \mathrm{~km}$, indicating that a totally difference receiver deployment should be considered in time-lapse seismic surveys.

For the qS-wave sensitivity, the sensitivity energy distributions for the VTI background model with both the ISO and TTI fracture zones have a fairly similar maximum concentration at approximately $3.5 \mathrm{~km}$. The sensitivity energies for the ISO background model are obviously separated into to two regions, one at approximately 2.5 to $3.0 \mathrm{~km}$ while the other at approximately $4.2 \mathrm{~km}$, which again indicates a necessity to consider the properties of background models and target monitoring zones when designing optimal time-lapse seismic surveys.

The isotropic/anisotropic properties of the background model and the fracture zone are also important for VSP receiver deployment. Figs. 6a and b show the $\mathrm{qP}$-wave sensitivity energies with respect to the elasticity parameter $C_{11}$ in the three VSP wells, while Figs. $6 \mathrm{c}$ and $\mathrm{d}$ show the $\mathrm{qS}$-wave sensitivity energies with respect to the elasticity parameter $C_{11}$ in the three VSP wells. Figs. 6e-h show the corresponding energy distributions of $E_{q S}^{C_{55}}$. The fracture zone is TTI in all the panels of Fig. 6. The background is VTI in Panels a, c, e and g in Fig. 6, while ISO in Panels b, d, f, h in Fig. 6.

Figs. $6 \mathrm{c}$ and $\mathrm{d}$ show the energy $E_{q S}^{C_{11}}$ in the VTI background model and the ISO background model, respectively. In both cases, the energy $E_{q S}^{C_{11}}$ in Well 2 has a maximum concentration at the bottom of the well, while has a maximum concentration at the depth of approximately $1.2 \mathrm{~km}$ in Well 3 . In Well 1 , the maximum energy concentration is obviously shallower in the VTI background model compared with that in the ISO background model.

Similarly, Figs. 6e and f depict the energy $E_{q P}^{C_{55}}$ in the VTI background model and the ISO background model, respectively. It is evident that the difference in the anisotropic property of the background model significantly alters the energy concentration in all the three wells. For instance, in Well 3, the energy concentration reaches the maximum at the depth of approximately $1.25 \mathrm{~km}$ in the VTI background model, while at the depth of approximately $0.9 \mathrm{~km}$ in the ISO background model, with a wider distribution.

It is evident that although Wells 2 and 3 are both close to the target monitoring fracture zone, the accumulated sensitivity energies in these two VSP wells are fairly different for both the $\mathrm{qP}$ - and the $\mathrm{qS}$-sensitivities because of the different background models. In different background models, the energy concentration in Well 1 can also vary significantly.

\section{Conclusions}

We have developed a new method for optimal design of cost-effective time-lapse seismic surveys for monitoring subsurface reservoirs in anisotropic media. We numerically model anisotropic elastic-wave sensitivity propagation, and use the spatial distributions 


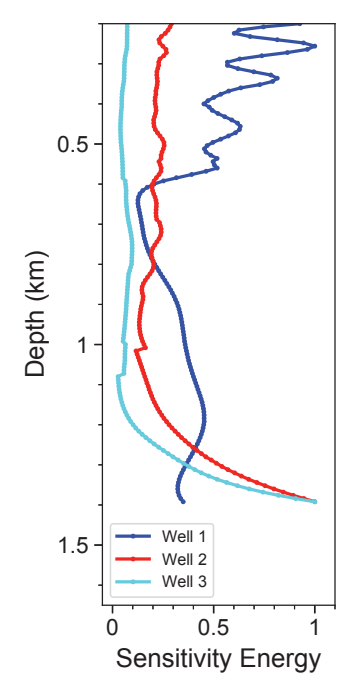

(a)

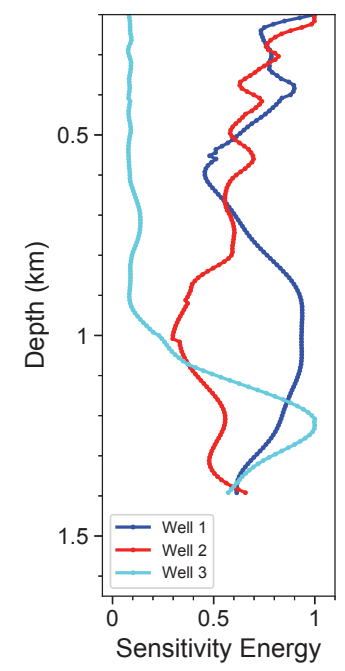

(e)

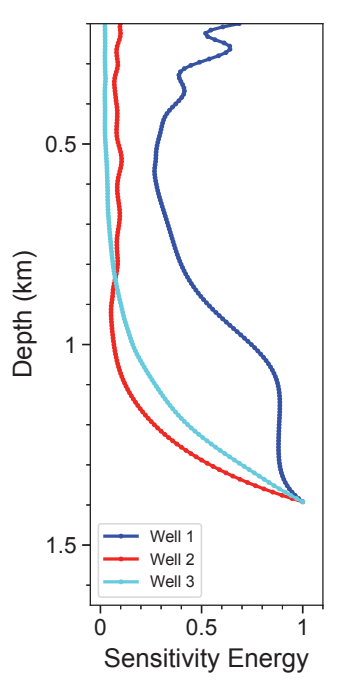

(b)

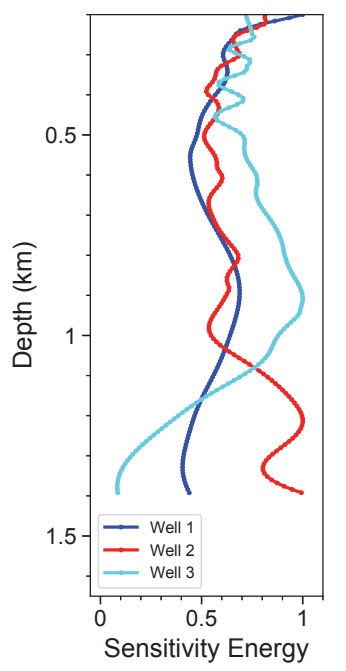

(f)

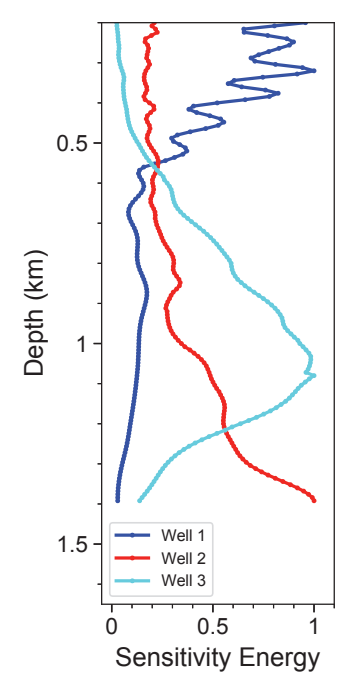

(c)

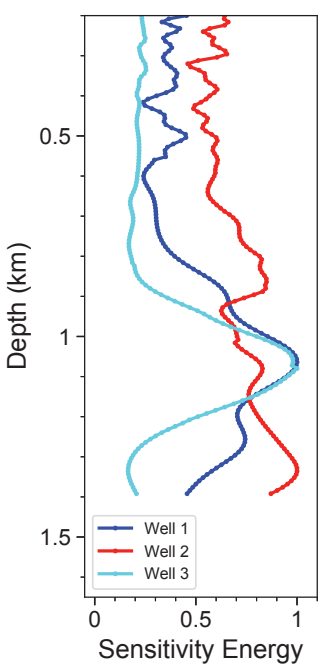

(g)

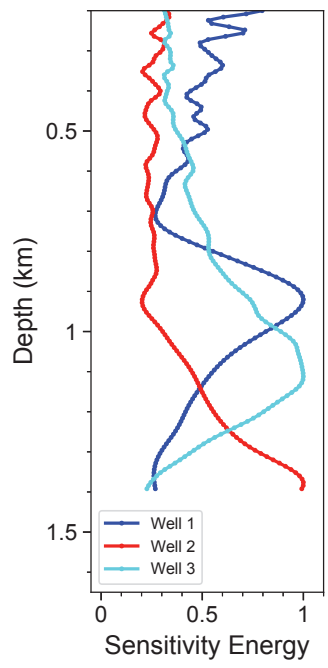

(d)

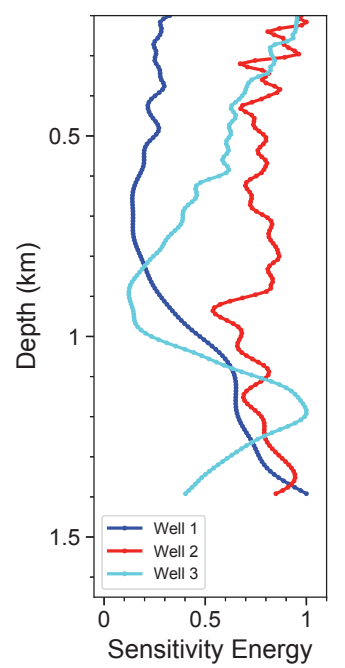

(h)

Figure 6: The qP- $(\mathrm{a}, \mathrm{b})$ and $\mathrm{qS}$-wave $(\mathrm{c}, \mathrm{d})$ sensitivity energies with respect to the elasticity parameter $C_{11}$ in three VSP wells and the $q P-(e, f)$ and $q S$-wave $(g, h)$ sensitivity energies with respect to the elasticity parameter $C_{55}$ in three VSP wells. The fracture zone is TTI in all the panels. The background is VTI in Panels $(a, c, e, g)$ and ISO in Panels (b, d, f, h).

of the $\mathrm{qP}$ - and $\mathrm{qS}$-wave sensitivity energies with respect to various elasticity parameters to determine the best receiver locations for time-lapse seismic survey design. We achieve accurate separation of the $\mathrm{qP}$ - and $\mathrm{qS}$-wave sensitivities in anisotropic media using a low-rank decomposition approach in the hybrid time and frequency domain. We verify the efficacy of our method using a modified Hess elastic model. Our numerical results 
show that the isotropic/anisotropic properties of the target monitoring fracture zone and the background model may lead to significantly different spatial distributions of elasticwave sensitivity energies in space. Therefore, it is important to properly account for the characteristics of anisotropy properties of target monitoring regions and the background model for optimal design of cost-effective time-lapse seismic surveys.

\section{Acknowledgments}

This work was supported by the U.S. Department of Energy (DOE) through the Los Alamos National Laboratory (LANL), which is operated by Triad National Security, LLC, for the National Nuclear Security Administration (NNSA) of U.S. DOE under Contract No. $89233218 \mathrm{CNA} 000001$. This research used resources provided by the LANL Institutional Computing Program, which is supported by the U.S. DOE NNSA under Contract No. 89233218CNA000001. We thank the Hess Corporation for making the Hess VTI model available.

\section{References}

[1] G. C. Alves, A. Bulco, D. M. S. Filho, C. E. Theodoro, and L. A. Santos. Target oriented approach for illumination analysis using wave equation via FDM. SEG Technical Program Expanded Abstracts, pages 181-185, 2009.

[2] G. Bear, C.-P. Lu, R. Lu, D. Willen, and I. Watson. The construction of subsurface illumination and amplitude maps via ray tracing. The Leading Edge, 19(7):726-728, 2000.

[3] J. M. Carcione. Wave Fields in Real Media: Wave Propagation in Anisotropic, Anelastic, Porous and Electromagnetic Media (Third Edition). Elsevier, Amsterdam, Netherlands, 2015.

[4] J. Cheng and S. Fomel. Fast algorithms for elastic-wave-mode separation and vector decomposition using low-rank approximation for anisotropic media. Geophysics, 79(4):C97-C110, 2014.

[5] A. Curtis. Theory of model-based geophysical survey and experimental design. The Leading Edge, 23(11):1112-1117, 2004.

[6] H. Denli and L. Huang. Elastic-wave sensitivity propagation. Geophysics, 75(3):T83-T97, 2010.

[7] K. Gao and L. Huang. An improved rotated staggered-grid finite-difference method with fourth-order temporal accuracy for elastic-wave modeling in anisotropic media. Journal of Computational Physics, 350:361 - 386, 2017.

[8] V. Grechka and M. Kachanov. Effective elasticity of rocks with closely spaced and intersecting cracks. Geophysics, 71(3):D85-D91, 2006.

[9] J. A. Hudson. Overall properties of a cracked solid. Mathematical Proceedings of the Cambridge Philosophical Society, 88(02):371-384, 1980.

[10] J. A. Hudson. Wave speeds and attenuation of elastic waves in material containing cracks. Geophysical Journal of the Royal Astronomical Society, 64(1):133-150, 1981.

[11] R. Laurain, L. J. Gelius, V. Vinje, and I. Lecomte. A review of 3D illumination studies. Journal of Seismic Exploration, 13:17-37, 2004. 
[12] E. Liu, J. Hudson, and T. Pointer. Equivalent medium representation of fractured rock. Journal of Geophysical Research, 105(B2):2981-3000, 2000.

[13] D. Muerdter and D. Ratcliff. Understanding subsalt illumination through ray-trace modeling, part 1: Simple 2-d salt models. The Leading Edge, 20(6):578-594, 2001.

[14] C. M. Sayers. Stress-dependent elastic anisotropy of sandstones. Geophysical Prospecting, 50(1):85-95, 2002.

[15] C. M. Sayers. Sensitivity of time-lapse seismic to reservoir stress path. Geophysical Prospecting, 54(3):369-380, 2006.

[16] C. M. Sayers. Seismic characterization of reservoirs containing multiple fracture sets. Geophysical Prospecting, 57(2):187-192, 2009.

[17] C. M. Sayers. Geophysics under stress: geomechanical applications of seismic and borehole acoustic waves. Number 13 in 2010 Distinguished Instructor Short Course. Society of Exploration Geophysicists and European Association of Geoscientists \& Engineers, 2010.

[18] C. M. Sayers and L. D. den Boer. Characterizing production-induced anisotropy of fractured reservoirs having multiple fracture sets. Geophysical Prospecting, 60(5):919-939, 2012.

[19] M. Schoenberg. Elastic wave behavior across linear slip interfaces. Journal of the Acoustical Society of America, 68:1516-1521, 1980.

[20] M. Schoenberg and C. M. Sayers. Seismic anisotropy of fractured rock. Geophysics, 60(1):204211, 1995.

[21] X. Shang and L. Huang. Optimal designs of time-lapse seismic surveys for monitoring $\mathrm{CO}_{2}$ leakage through fault zones. International Journal of Greenhouse Gas Control, 10:419 - 433, 2012.

[22] L. Sirgue, J. Etgen, and U. Albertin. 3D frequency domain waveform inversion using time domain finite difference methods. Expanded Abstracts of 70th EAGE Conference \& Exhibition, Rome, Italy, 2008.

[23] L. Thomsen. Elastic anisotropy due to aligned cracks in porous rock. Geophysical Prospecting, 43(6):805-829, 1995.

[24] I. Tsvankin. Seismic signatures and analysis of reflection data in anisotropic media. Elsevier Science Publ. Co., Inc., 2nd edition, 2005.

[25] X.-B. Xie, S. Jin, and R.-S. Wu. Wave-equation-based seismic illumination analysis. Geophysics, 71(5):S169-S177, 2006. 